\title{
Identification of a novel IncRNA GABPB1-IT1 that is downregulated and predicts a poor prognosis in non-small cell lung cancer
}

\author{
JIANJIANG XIE, GUIJING XIE, QUHAI CHEN, ZIXUN XU, \\ WENJIE BAI and MINDONG CHEN \\ Department of Thoracic Surgery, Guangzhou First People's Hospital, The Second Affiliated Hospital \\ of South China University of Technology, Guangzhou, Guangdong 510180, P.R. China
}

Received May 27, 2018; Accepted April 15, 2019

DOI: $10.3892 / \mathrm{ol} .2019 .10357$

\begin{abstract}
Lung cancer is the leading cause of cancer-associated mortality worldwide. Previous studies have demonstrated that long non-coding RNAs (lncRNAs) serve important roles in diverse biological processes. However, the molecular function and prognostic value of the majority of lncRNAs in non-small cell lung cancer (NSCLC) remain unknown. The present study investigated the expression of the lncRNA GABPB1 intronic transcript (GABPB1-IT1) in NSCLC tissues using publicly available databases. Subsequently, protein-protein interaction (PPI) and co-expression networks were constructed to identify key targets of 1ncRNA GABPB1-IT1. Furthermore, gene ontology (GO) and Kyoto Encyclopedia of Genes and Genomes (KEGG) pathway analysis were performed to investigate the potential roles of 1ncRNA GABPB1-IT1. The current study identified that the expression of GABPB1-IT1 was significantly downregulated in NSCLC samples compared with normal samples. Furthermore, the expression levels of GABPB1-IT1 were lower in high grade NSCLC samples compared with low grade NSCLC samples. Additionally, overexpression of GABPB1-IT1 in cancer samples was associated with improved survival of patients with NSCLC. GABPB1-IT1 was revealed to be involved in the regulation of cell cycle-associated biological processes, including sister chromatid cohesion, mitotic nuclear division, DNA replication, chromosome segregation, G1/S transition of mitotic cell cycle, mitotic cytokinesis and cell division. Finally, a GABPB1-IT1-associated protein-protein interaction network was constructed for NSCLC. To the best of our knowledge, the
\end{abstract}

Correspondence to: Dr Mindong Chen, Department of Thoracic Surgery, Guangzhou First People's Hospital, The Second Affiliated Hospital of South China University of Technology, 1 Pan Fu Road, Guangzhou, Guangdong 510180, P.R. China

E-mail: cmd628@yeah.net

Key words: long non-coding RNA, non-small cell lung cancer, protein-protein interaction analysis, co-expression network present study was the first to demonstrate that GABPB1-IT1 is associated with the prognosis of NSCLC. The current study provides useful information to assist with the investigation of potential candidate biomarkers for diagnosis, prognosis and drug targets for NSCLC.

\section{Introduction}

Long non-coding RNAs (lncRNAs) are a class of non-coding RNAs, which consist of $>200$ nucleotides that are not translated into proteins $(1,2)$. Previous studies have revealed that lncRNAs serve important roles in various types of human disease by regulating gene transcription, epigenetic modifications and cell proliferation $(3,4)$. In previous studies, an abnormal expression of lncRNAs has been observed in different types of cancer, including lung, liver, gastric, prostate and breast cancer $(5,6)$. Notably, IncRNAs have been demonstrated to possess significant potential prognostic value for cancer. For example, it has been reported that the sensitivity of the lncRNA prostate cancer antigen 3 is higher compared with prostate-specific antigen for the urinary detection of prostate cancer (7). Decreased expression of the lncRNA SLC7A11-AS1 predicted a poor prognosis in gastric cancer (8). Higher LINC00959 expression has been negatively associated with Tumor-Node-Metastasis (TNM) stage, distant metastasis and lymphatic metastasis, and negatively correlated with improved prognosis in colorectal cancer (9). Therefore, investigation of cancer-associated lncRNAs could provide novel insights that assist with the identification of biomarkers for cancer.

Lung cancer, including small cell lung carcinoma and non-small cell lung carcinoma (NSCLC), is the leading cause of cancer-associated mortality worldwide in 2018 (10). In the USA, lung cancer was estimated to account for $26 \%$ of all cancer-associated mortalities in 2017 (11). In China, $\sim 730,000$ new cases of lung cancer were diagnosed in 2015 and 610,000 lung-cancer associated mortalities were reported (12). In the USA, despite improvements in lung cancer treatment, the 5-year survival rate for lung cancer remains as low as $\sim 15 \%$ in 2018 (13). NSCLC is the predominant type of lung cancer and accounts for $80 \%$ of lung cancer cases (14). A number of IncRNAs have been identified to be associated with NSCLC 
progression. For example, the lncRNA small nucleolar RNA host gene 1 promotes NSCLC progression via an upregulation of metadherin by sponging microRNA (miR)-145-5p (15). The IncRNA X-inactive specific transcript has been reported to promote the transforming growth factor- $\beta$-induced epithelial-mesenchymal transition by regulating the miR-367/141-zinc finger E-box binding homeobox 2 axis in NSCLC (16). In addition, Lai et al (17) identified that the IncRNA myocardial infarction associated transcript promotes NSCLC proliferation and metastasis via matrix metallopeptidase 9 activation. However, to the best of our knowledge, the molecular function and prognostic value of the majority of lncRNAs in NSCLC remain unknown.

The present study investigated the expression of the IncRNA GABPB1 intronic transcript (GABPB1-IT1) in NSCLC tissues using publicly available databases. Subsequently, the associations between GABPB1-IT1 expression and the characteristics of patients with NSCLC were evaluated. Protein-protein interaction (PPI) and co-expression networks were constructed to identify key targets of GABPB1-IT1. Furthermore, gene ontology (GO) and Kyoto Encyclopedia of Genes and Genomes (KEGG) pathway analyses were performed to investigate the potential roles of GABPB1-IT1. The present study provides useful information that may assist with the investigation of potential candidate biomarkers for diagnosis, prognosis and drug targets for NSCLC.

\section{Materials and methods}

Microarray data and data preprocessing. The gene expression data of lung adenocarcinoma (LUAD) and lung squamous cell carcinoma (LUSC) were downloaded from The Cancer Genome Atlas (TCGA; https://cancergenome.nih.gov/) database. TCGA data was downloaded from cBioPortal (http://cbioportal.org). The methods of biospecimen procurement, RNA isolation and RNA sequencing were performed as previously described by the Cancer Genome Atlas Research Network (18). Patient clinical features, including age at diagnosis, overall survival time, disease-free survival time, pathological tumor (T) stage and node $(\mathrm{N})$ stage, were retrospectively obtained from patient records. All the patients were staged using the 2009 TNM classification of the American Joint Committee on Cancer/International Union Against Cancer (19).

Survival analysis. Kaplan-Meier plotter (KM plotter) is a web tool that predicts the effect of genes on survival (kmplot.com/analysis/index.php? $\mathrm{p}=$ service \&cancer=lung version: 2016.04.08) (20). By entering the gene of interest into the website, patients were divided into two groups according to the expression level of the gene and the survival rate was statistically analyzed. The hazard ratio with $95 \%$ confidence intervals and the log-rank P-value were calculated and presented for each analysis.

Co-expression network construction and analysis. The Pearson correlation coefficient of GABPB1-IT1-mRNA pairs was calculated according to the expression value. The co-expressed GABPB1-IT1-mRNA pairs with a Pearson correlation coefficient $\geq 0.30$ were selected to generate a co-expression network using Cytoscape software (version 3.4.0; www.cytoscape.org/). According to this cut-off value, $5 \%(896 / 18,603)$ of the mRNAs were included in this co-expression network. The Cytoscape MCODE plug-in was used for visualization of the co-expression networks.

Construction of a PPI network and module analysis. To predict protein interactions, including physical and functional associations, the present study used the Search Tool for the Retrieval of Interacting Genes (STRING) database (21) to construct a PPI network of GABPB1-IT1 co-expressing mRNAs. The associations of the proteins encoded by the DEGs were analyzed using STRING and a combined score $>0.4$ was used as the cut-off criterion. In addition, Cytoscape software 3.4.0 (http://cytoscape.org/download_old_versions.html) was used for visualization of the PPI networks (22). Following the construction of the PPI network, a module analysis of the network was performed using the Cytoscape MCODE plug-in with the following criterion: Degree cut-off $\geq 2$ (23). Additionally, Network Analyzer (a plug-in for Cytoscape) was used to compute the basic properties of the PPI network, including the average clustering coefficient distribution, closeness centrality, average neighborhood connectivity, node degree distribution, shortest path length distribution and topological coefficients (24).

$G O \& K E G G$ pathway analysis. To identify functions of the DEGs in NSCLC, GO functional enrichment analysis was performed for the following three categories: Biological process, cellular component and molecular function. In addition, KEGG pathway enrichment analysis was performed to identify pathways enriched in NSCLC using the database for annotation, visualization and integrated discovery (DAVID) (https://david.ncifcrf.gov/). $\mathrm{P}<0.05$ was considered to indicate a statistically significant result.

Statistical analysis. All statistical analyses were performed using R software version 3.3 (Detail in https://www.r-project. org) and GraphPad Prism 6 software (GraphPad Software, Inc. La Jolla, CA, USA). All numerical data are presented as the mean \pm standard deviation of a minimum of three determinations. Statistical comparisons between two groups of normalized data were performed using a Mann-Whitney $\mathrm{U}$ test. Differences among multiple groups were analyzed by one-way analysis of variance (ANOVA) following Bonferroni post hoc test. $\mathrm{P}<0.05$ was considered to indicate a statistically significant difference.

\section{Results}

lncRNA GABPB1-IT1 is downregulated in NSCLC. The expression levels of GABPB1-IT1 in NSCLC were analyzed using TCGA data from cBioPortal (www.cbioportal.org/). As presented in Fig. 1A, analysis of the TCGA LUAD dataset revealed that the expression level of GABPB1-IT1 was significantly lower in tumor tissues compared with normal tissues. To further validate this result, the TCGA LUSC dataset was analyzed, which also revealed a significantly lower expression level of GABPB1-IT1 in tumor samples compared with normal tissues (Fig. 1C).

To further evaluate the possible prognostic value of GABPB1-IT1, LUAD and LUSC RNA-seq data from TCGA 

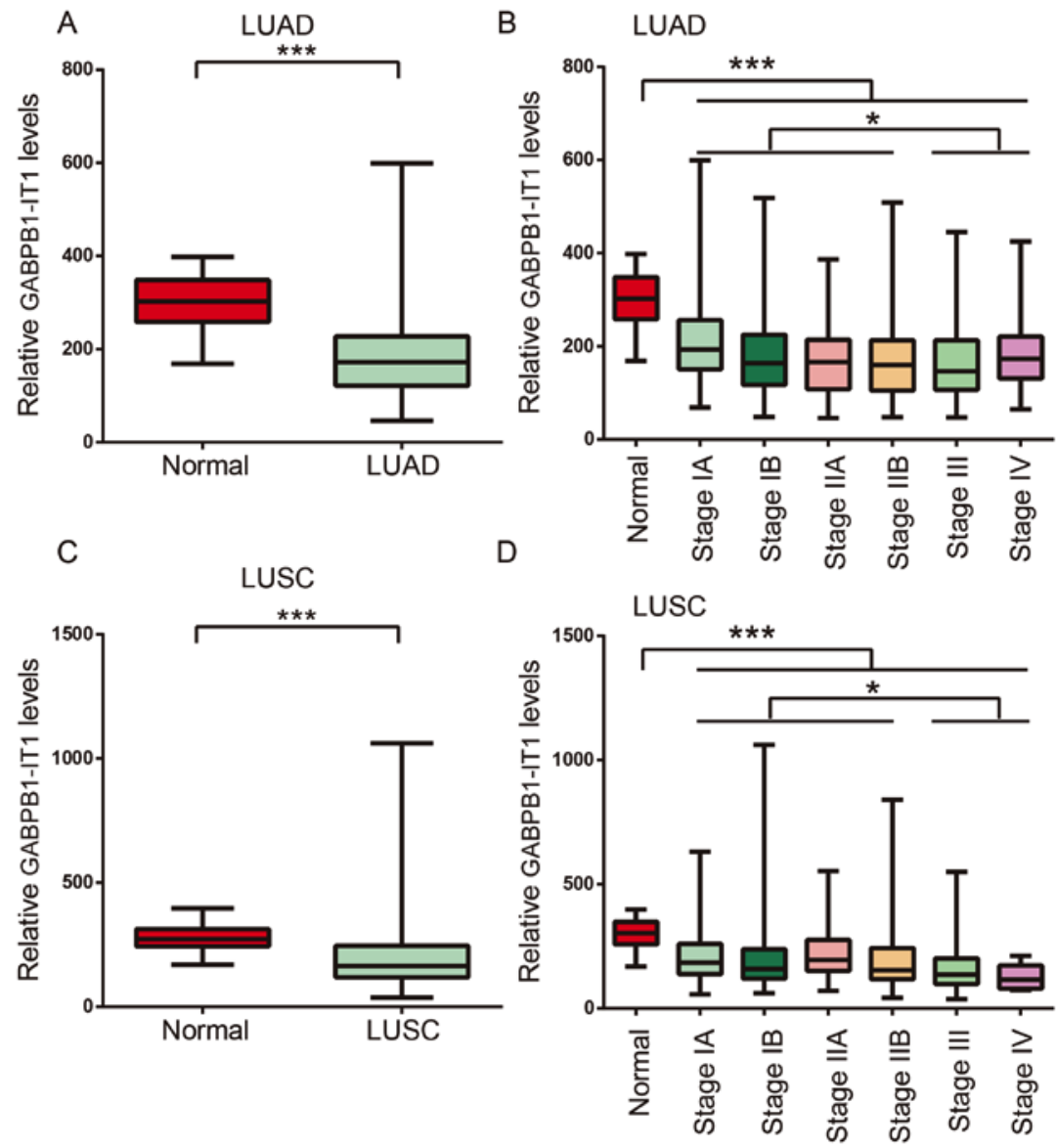

Figure 1. Long non-coding RNA GABPB1-IT1 is downregulated in non-small cell lung cancer. (A) GABPB1-IT1 expression levels in LUAD tissues compared with normal LUAD tissues in TCGA LUAD dataset. (B) GABPB1-IT1 expression levels in LUAD different stage tissues compared with normal LUAD tissues. (C) GABPB1-IT1 expression levels in LUAD tissues compared with normal LUSC tissues in TCGA LUSC dataset. (D) GABPB1-IT1 expression levels in LUSC different stage tissues compared with normal LUSC tissues. Data are presented as the mean \pm standard deviation. " $\mathrm{P}<0.05$ and ${ }^{* * * *} \mathrm{P}<0.001$, as indicated. GABPB1-IT1, GABPB1 intronic transcript; TCGA, The Cancer Genome Atlas; LUAD, lung adenocarcinoma; LUSC, lung squamous cell carcinoma.

were analyzed. It was identified that GABPB1-IT1 expression was significantly lower in patients with stage I $(\mathrm{P}<0.001)$, II $(\mathrm{P}<0.001)$, III $(\mathrm{P}<0.001)$ and IV $(\mathrm{P}<0.001)$ NSCLC compared with the normal controls (Fig. 1B and D). Furthermore, it was revealed that the expression level of GABPB1-IT1 was significantly lower in high grade NSCLC samples (stages III and IV) compared with low grade NSCLC samples (stages I and II; Fig. 1B and D).

Downregulation of GABPB1-IT1 predicts a poor prognosis in $N S C L C$. Kaplan-Meier analysis was performed to determine whether GABPB1-IT1 expression was significantly associated with disease-free survival and overall survival time in patients with NSCLC by analyzing TCGA LUAD dataset. The median GABPB1-IT1 expression level in NSCLC tissues was used as the cut-off value to divide all cases into high and low GABPB1-IT1 groups. The results revealed that the overall survival and disease-free survival rates were significantly higher in the GABPB1-IT1-high group compared with the GABPB1-IT1-low group (Fig. 2A and B). These results indicate that GABPB1-IT1 expression could serve as a biomarker to predict the outcome of NSCLC.

Subsequently, the Kaplan-Meier plotter database was used to comprehensively analyze the association of lncRNA GABPB1-IT1 expression with the survival rates in
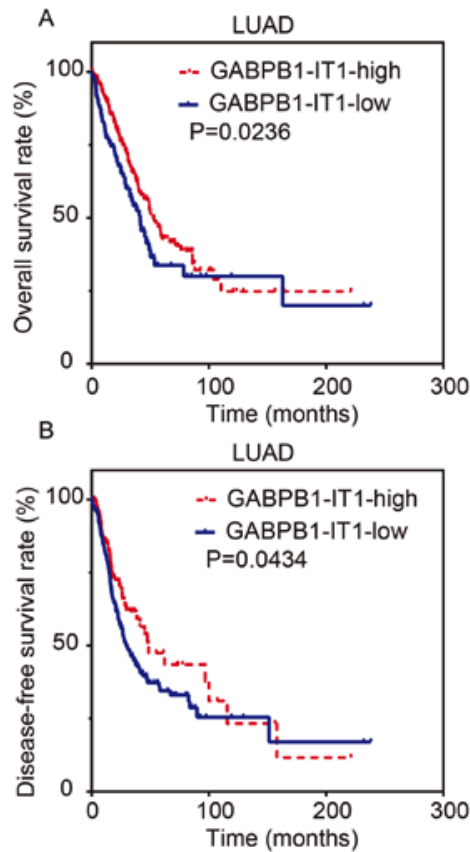

Figure 2. Prognostic value of GABPB1-IT1 in non-small cell lung cancer. Kaplan-Meier analysis of the (A) overall survival and (B) disease-free survival rates in patients with high GABPB1-IT1 expression compared with patients with low GABPB1-IT1 expression. GABPB1-IT1, GABPB1 intronic transcript; LUAD, lung adenocarcinoma. 

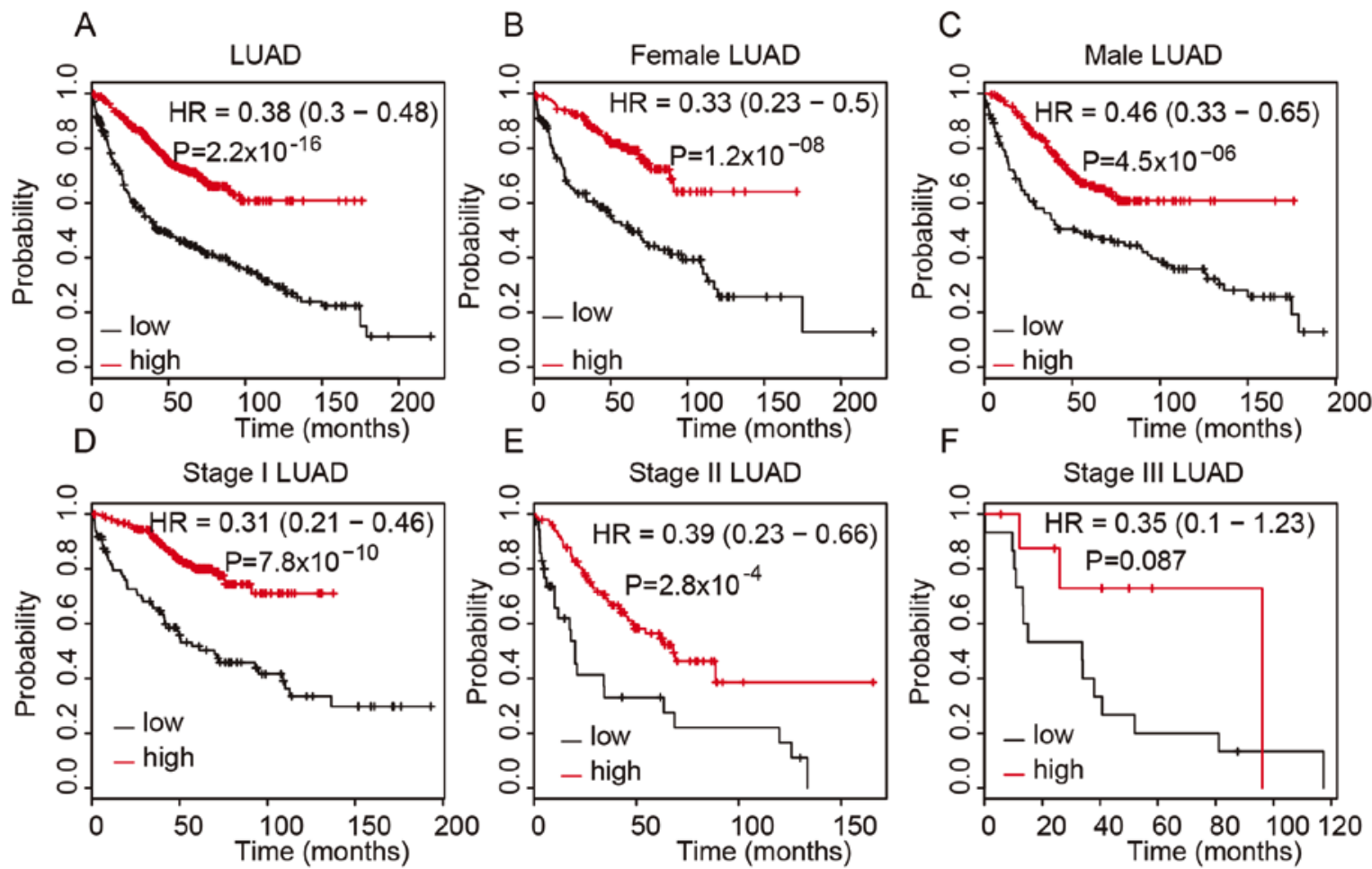

Figure 3. Downregulation of GABPB1-IT1 predicts a poor prognosis in LUAD tissues Kaplan-Meier plotter database analysis of the overall survival and disease-free survival times in patients with high GABPB1-IT1 expression compared with patients with low GABPB1-IT1 expression for (A) all cases of LUAD, (B) cases of LUAD in females, (C) cases of LUAD in males, (D) stage ILUAD, (E) stage II LUAD and (F) stage III LUAD. GABPB1-IT1, GABPB1 intronic transcript; LUAD, lung adenocarcinoma; NSCLC, non-small cell lung cancer.

various types of LUAD. As presented in Fig. 3, the overall survival time was lower in the GABPB1-IT1-low groups compared with the GABPB1-IT1-high groups for all LUAD cases, female LUAD cases and male LUAD cases (Fig. 3A-C) and for stages I, II and III LUAD cases (Fig. 3D-F).

Co-expression network analysis and enrichment analysis of GABPB1-IT1 in NSCLC. The present study performed co-expression network analysis to predict the potential role of GABPB1-IT1 in NSCLC by calculating the Pearson correlation coefficient of GABPB1-IT1-target pairs. A Pearson correlation coefficient $\geq 0.30$ was selected as the cut-off value to identify potential GABPB1-IT1 targets in NSCLC. The co-expression network, constructed using Cytoscape, is presented in Fig. 4. In total, the network included 896 mRNAs.

DAVID was used to perform GO and pathway analysis of the co-expressing genes of GABPB1-IT1. GO analysis revealed that GABPB1-IT1 was predominantly involved in 'sister chromatid cohesion', 'mitotic nuclear division', 'DNA replication', 'regulation of transcription', 'positive regulation of protein localization to Cajal body', 'chromosome segregation', 'G1/S transition of mitotic cell cycle', 'mitotic cytokinesis', 'cell division' and 'sarcomere organization' (Fig. 5A). Furthermore, the molecular functions of GABPB1-IT1 were associated with 'nucleic acid binding', 'DNA binding', 'ATP binding', 'damaged DNA binding' and 'metal ion binding' (Fig. 5B).

KEGG pathway analysis revealed that GABPB1-IT1 was primarily enriched in pathways associated with 'Cell cycle',
'Fanconi anemia pathway', 'One carbon pool by folate', 'RNA transport', 'Proteasome', 'Base excision repair', 'Primary immunodeficiency', 'Oocyte meiosis', 'DNA replication' and 'Arachidonic acid metabolism' (Fig. 5C).

Construction of a GABPB1-IT1-associcated PPI network. Based on the STRING database, a PPI network was constructed to predict the interaction associations among 896 GABPB1-IT1 co-expressed proteins in NSCLC (combined score $>0.4$ ). Following the construction of the PPI network, a module analysis of the network was performed using the MCODE plugin (degree cut-off $\geq 2$ ).

Three hub-networks associated with IncRNA GABPB1-IT1 were identified. As presented in Fig. 6, one hub-network contained 46 nodes, including DEPDC1, CCNA2, SKA3, TPX2, HMMR, FOXM1, NCAPG2, CENPN, CDKN3, MCM10, ESPL1, RAD51AP1, MAD2L1, SHCBP1, CHEK1, EXO1, CDT1 and FEN1, and 964 edges (Fig. 6A). Another hub-network contained 17 nodes, including SRSF5, ACLY, ALYREF, TARS, SRSF11, TCP1, CCT5, EIF4A3, MTHFD1L, SF3B2, EFTUD2, RBM5, PCF11, CCT7, MAT2A, TMX3 and STIP1, and 67 edges (Fig. 6B). The final hub-network contained 11 nodes, including C3, GNAQ, GPR55, GRM6, GABBR1, ADRA2C, GNRH1, LTB4R2, GNRHR2, ADCY4 and NPFF, and 28 edges (Fig. 6C).

\section{Discussion}

Lung cancer is the leading cause of cancer-associated mortality worldwide and the 5-year survival rate for lung 


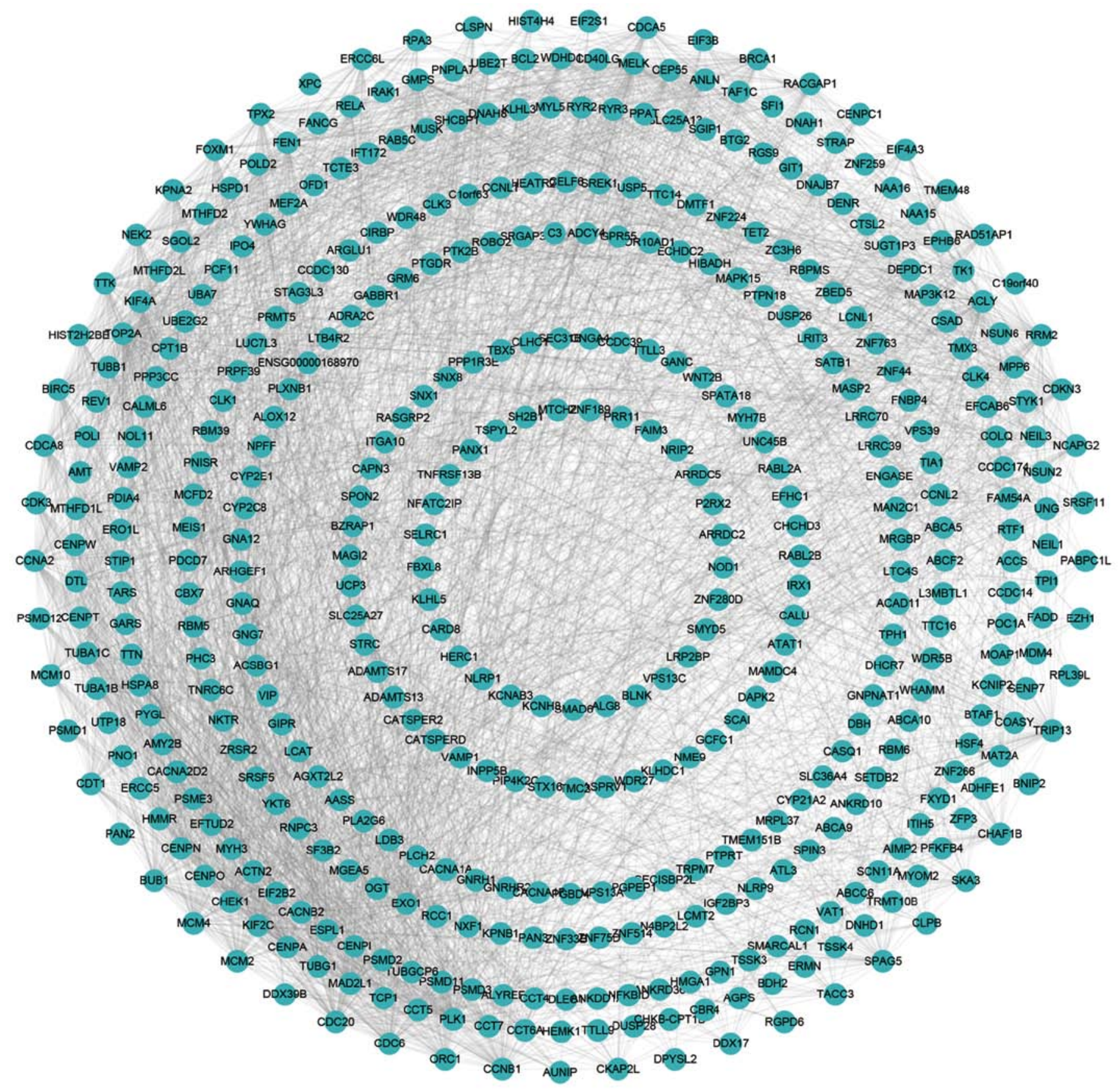

Figure 4. Co-expression network analysis of GABPB1-IT1 in NSCLC. Co-expression network analysis revealed 896 mRNAs as potential targets of GABPB1-IT1 in NSCLC. GABPB1-IT1, GABPB1 intronic transcript; NSCLC, non-small cell lung cancer.

cancer is as low as $\sim 15 \%$ (13). In China, $\sim 730,000$ new cases of lung cancer were diagnosed in 2015 and $>610,000$ lung cancer-associated mortalities were reported (12). Previous studies have demonstrated that IncRNAs are associated with lung cancer prognosis and a number of lncRNAs have been reported to be dysregulated in lung cancer. For example, Luo et al (25) identified that the lncRNAs RP5-821D11.7, APCDD1L-AS1 and RP11-277P12.9 were associated with the prognosis of lung squamous cell carcinoma. Overexpression of lncRNA MetaLnc9 in human NSCLC samples has been correlated with a poor prognosis, and has been revealed to promote migration and invasion of NSCLC cells in vitro, and enhance lung metastasis formation in vivo (26). The present study demonstrated that GABPB1-IT1 expression was significantly downregulated in LUSC and LUAD samples compared with normal samples by analyzing TCGA datasets. Furthermore, it was identified that GABPB1-IT1 expression was lower in high grade NSCLC samples compared with low grade NSCLC samples. Using Kaplan-Meier analysis, the present study revealed that higher expression levels of GABPB1-IT1 in NSCLC were associated with an improved survival time in different types and stages of LUAD, including female, male, stage I, stage II and stage III LUAD samples. To the best of our knowledge, the current study is the first to report that GABPB1-IT1 may serve as a biomarker for NSCLC. 
A

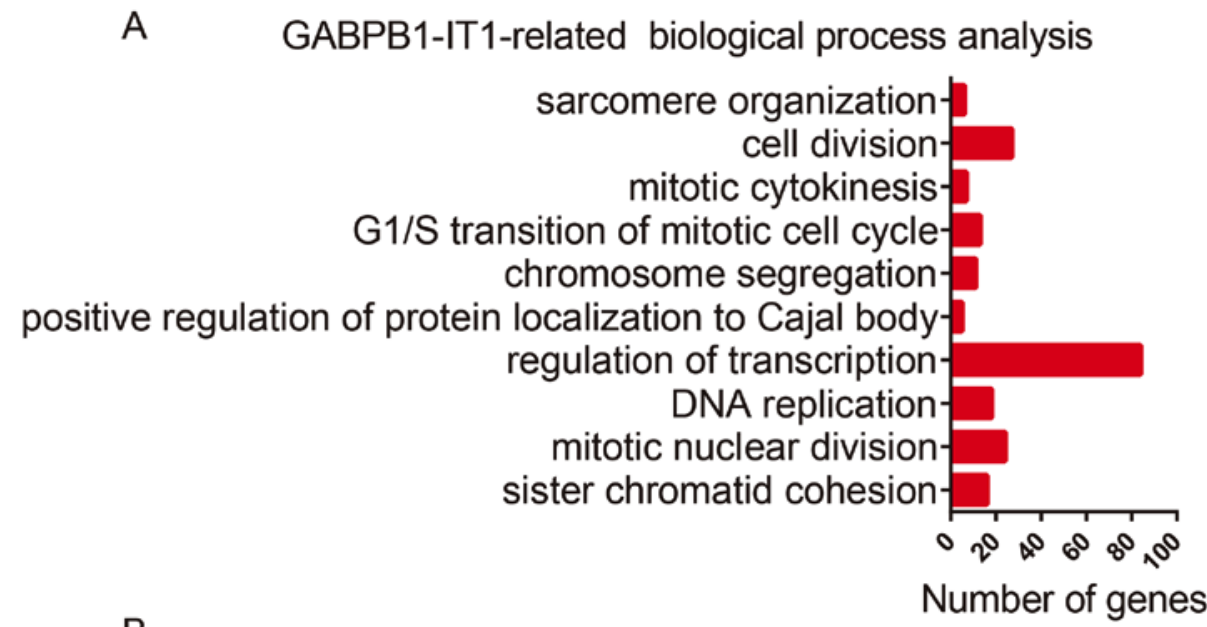

B

GABPB1-IT1-related molecular function analysis

nucleotide binding
methylenetetrahydrofolate dehydrogenase activity
formate-tetrahydrofolate ligase activity-
methenyltetrahydrofolate cyclohydrolase activity-
single-stranded DNA binding
metal ion binding
damaged DNA binding
ATP binding
DNA binding
nucleic acid binding

Number of genes

GABPB1-IT1-related KEGG pathway analysis

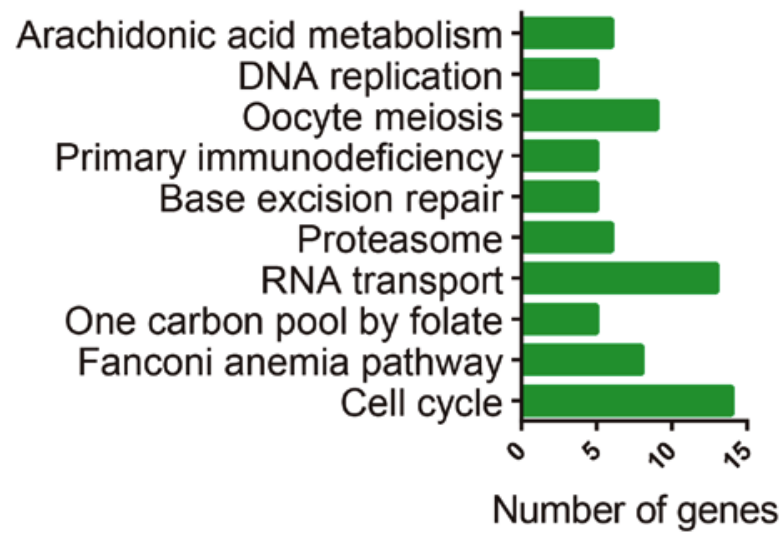

Figure 5. Enrichment analysis of GABPB1-IT1 in non-small cell lung cancer. Gene ontology functional enrichment analysis was performed for (A) GABPB1-IT1-related biological process terms and (B) GABPB1-IT1-related molecular function terms. (C) GABPB1-IT1-related KEGG pathway analysis. GABPB1-IT1, GABPB1 intronic transcript; KEGG, Kyoto Encyclopedia of Genes and Genomes; ATP, adenosine 5'-triphosphate.

Previous studies have revealed that $\operatorname{lncRNAs}$ serve important roles in various types of human disease by regulating expression of protein-coding genes through transcriptional, post-transcriptional and posttranslational regulation. For example, the lncRNA small nucleolar RNA host gene 20 has been demonstrated to promote NSCLC cell proliferation and migration by epigenetically silencing $\mathrm{p} 21$ expression (27). The lncRNA long intergenic non-protein coding RNA 673 has been identified to promote NSCLC metastasis by binding with enhancer of zeste 2 polycomb 2 and causing epigenetic silencing of homeoboz A5 (28). The present study revealed that GABPB1-IT1 may serve as a biomarker for NSCLC; however, the functional roles of GABPB1-IT1 remain largely unknown. The current study performed co-expression analysis for GABPB1-IT1 and then used DAVID to perform GO and pathway analysis for the 


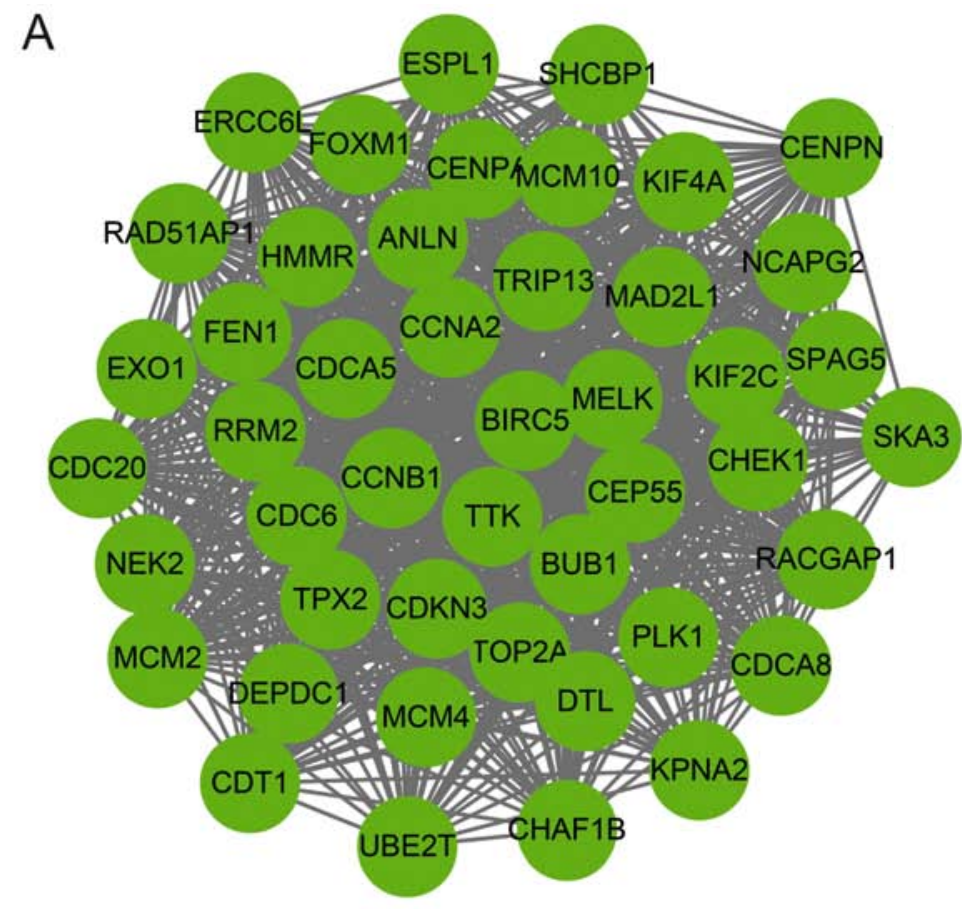

B

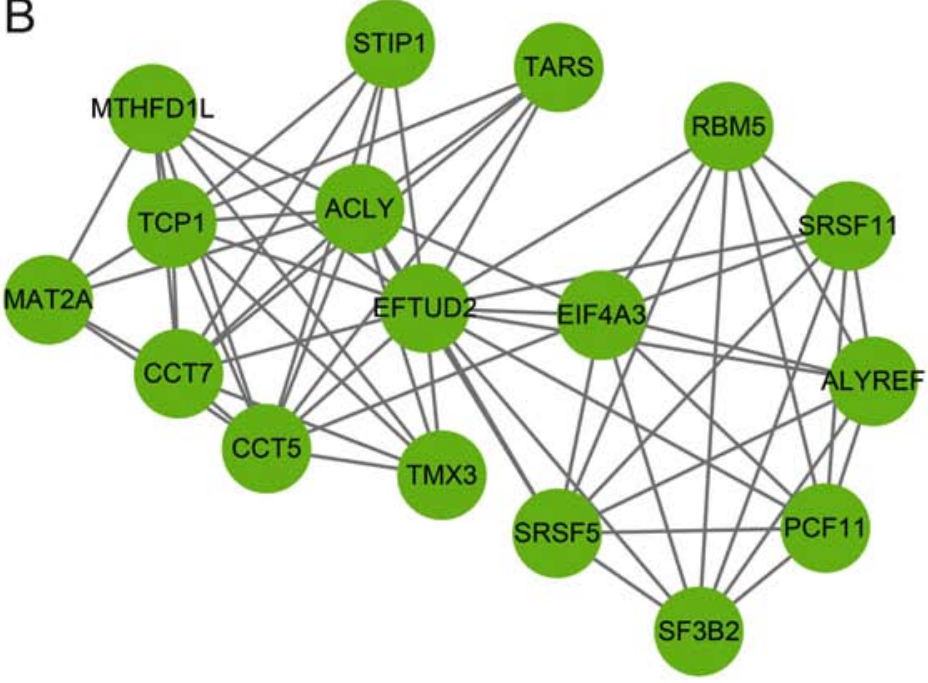

C

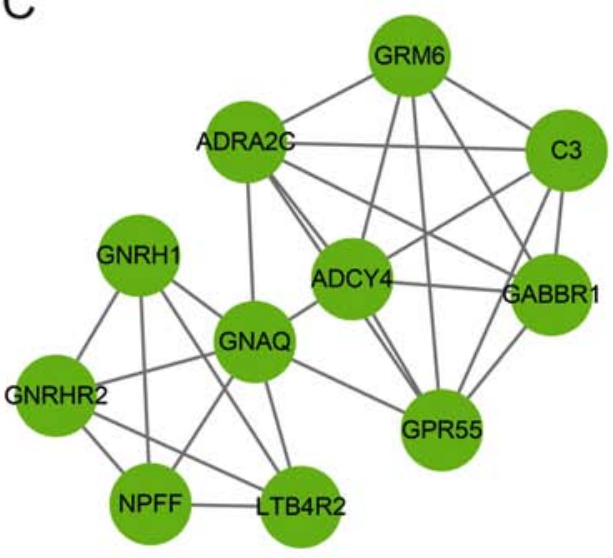

Figure 6. GABPB1-IT1-associated protein-protein interaction networks. (A) Hub-network 1 contained 46 nodes and 964 edges. (B) Hub-network 2 contained 17 nodes and 67 edges. (C) Hub-network 3 contained 11 nodes and 28 edges. GABPB1-IT1, GABPB1 intronic transcript.

co-expressed genes. GO analysis revealed that GABPB1-IT1 was predominantly involved in cell cycle and proliferation by regulating a number of cell cycle-associated biological processes, including 'sister chromatid cohesion', 'mitotic nuclear division', 'DNA replication', 'regulation of transcription', 'chromosome segregation', 'G1/S transition of mitotic cell cycle', 'mitotic cytokinesis and cell division'. Molecular functions showed GABPB1-IT1 was mainly involved in regulating DNA binding, suggested that GABPB1-IT1 may participate in the regulation of targets transcription. KEGG pathway analysis identified that GABPB1-IT1 was primarily enriched in pathways associated with 'Cell cycle', 'Fanconi anemia pathway', 'One carbon pool by folate', 'RNA transport', 'Proteasome', 'Base excision repair' and 'Primary immunodeficiency'. Furthermore, a GABPB1-IT1-associated
PPI network was constructed for NSCLC. Taken together, the results of the present study indicate that GABPB1-IT1 co-expressing genes were involved in regulating cell proliferation, such as MCM10 and CCNA2.

In conclusion, to the best of our knowledge, the present study is the first to report that GABPB1-IT1 is associated with the prognosis of NSCLC. Low GABPB1-IT1 expression levels were observed in NSCLC samples and were revealed to be associated with poor survival of patients with NSCLC. Furthermore, potential roles for GABPB1-IT1 in the regulation of the cell cycle were identified and a GABPB1-IT1-associated PPI network was constructed for NSCLC. In summary, the present study may assist with the identification of new therapeutic and prognostic targets for NSCLC. 


\section{Acknowledgements}

Not applicable.

\section{Funding}

No funding was received.

\section{Availability of data and materials}

All data generated or analyzed in this study are included in this manuscript.

\section{Authors' contributions}

JX and MC conceived and designed the study, and wrote, reviewed and revised the manuscript. JX, GX and QC obtained the data. JX, ZX and WB analyzed and interpreted the data. All authors read and approved the manuscript.

\section{Ethics approval and consent to participate}

Not applicable.

\section{Patient consent for publication}

Not applicable.

\section{Competing interests}

The authors declare that they have no competing interests.

\section{References}

1. Zamore PD and Haley B: Ribo-gnome: The big world of small RNAs. Science 309: 1519-1524, 2005.

2. Kornienko AE, Guenzl PM, Barlow DP and Pauler FM: Gene regulation by the act of long non-coding RNA transcription. BMC Biol 11: 59, 2013.

3. Wan X, Huang W, Yang S, Zhang Y, Pu H, Fu F, Huang Y, Wu H, $\mathrm{Li} \mathrm{T}$ and $\mathrm{Li} \mathrm{Y}$ : Identification of androgen-responsive lncRNAs as diagnostic and prognostic markers for prostate cancer. Oncotarget 7: 60503-60518, 2016.

4. Shi X, Sun M, Liu H, Yao Y and Song Y: Long non-coding RNAs: A new frontier in the study of human diseases. Cancer Lett 339: 159-166, 2013.

5. Zhang H, Chen Z, Wang X, Huang Z, He Z and Chen Y: Long non-coding RNA: A new player in cancer. J Hematol Oncol 6: 37, 2013.

6. Hauptman $\mathrm{N}$ and Glavač D: Long non-coding RNA in cancer. Int J Mol Sci 14: 4655-4669, 2013.

7. Ferreira LB, Palumbo A, de Mello KD, Sternberg C, Caetano MS, de Oliveira FL, Neves AF, Nasciutti LE, Goulart LR and Gimba ERP: PCA3 noncoding RNA is involved in the control of prostate-cancer cell survival and modulates androgen receptor signaling. BMC Cancer 12: 507, 2012.

8. Luo Y, Wang C, Yong P, Ye P, Liu Z, Fu Z, Lu F, Xiang W, Tan W and Xiao J: Decreased expression of the long non-coding RNA SLC7A11-AS1 predicts poor prognosis and promotes tumor growth in gastric cancer. Oncotarget 8: 112530-112549, 2017.

9. Sun ZQ, Chen C, Zhou QB, Liu JB, Yang SX, Li Z, Ou CL, Sun XT, Wang GX, Song JM, et al: Long non-coding RNA LINC00959 predicts colorectal cancer patient prognosis and inhibits tumor progression. Oncotarget 8: 97052-97060, 2017.
10. Sun S, Schiller JH and Gazdar AF: Lung cancer in Never-Smokers: A different disease. Nat Rev Cancer 7: 778-790, 2007.

11. Stewart B and Wild CP: World cancer report 2014. Health. 2017.

12. Chen W, Zheng R, Baade PD, Zhang S, Zeng H, Bray F, Jemal A, Yu XQ and He J: Cancer statistics in China, 2015. CA Cancer J Clin 66: 115-132, 2016.

13. Herbst RS, Morgensztern D and Boshoff C: The biology and management of non-small cell lung cancer. Nature 553: 446-454, 2018.

14. Brennan P, Hainaut $P$ and Boffetta P: Genetics of lung-cancer susceptibility. Lancet Oncol 12: 399-408, 2011.

15. Lu Q, Shan S, Li Y, Zhu D, Jin W and Ren T: Long noncoding RNA SNHG1 promotes non-small cell lung cancer progression by up-regulating MTDH via sponging miR-145-5p. FASEB J 32: 3957-3967, 2018

16. Li C, Wan L, Liu Z, Xu G, Wang S, Su Z, Zhang Y, Zhang C, Liu $X$ and Lei Z: Long non-coding RNA XIST promotes TGF- $\beta$-induced epithelial-mesenchymal transition by regulating miR-367/141-ZEB2 axis in non-small-cell lung cancer. Cancer Lett 418: 185-195, 2018.

17. Lai IL, Yang CA, Lin PC, Chan WL, Lee YT, Yen JC, Chang YS and Chang JG: Long noncoding RNA MIAT promotes non-small cell lung cancer proliferation and metastasis through MMP9 activation. Oncotarget 8: 98148-98162, 2017.

18. Cancer Genome Atlas Research Network, Kandoth C, Schultz N, Cherniack AD, Akbani R, Liu Y, Shen H, Robertson AG, Pashtan I, Shen R, et al: Integrated genomic characterization of endometrial carcinoma. Nature 497: 67-73, 2013.

19. Rami-Porta R, Ball D, Crowley J, Giroux DJ, Jett J, Travis WD, Tsuboi M, Vallieres E, Goldstraw P, Committee IS, et al: The IASLC lung cancer staging project: Proposals for the revision of the T descriptors in the forthcoming (seventh) edition of the TNM classification for lung cancer. J Thoracic Oncol 2: 593-602, 2007.

20. Győrffy B, Surowiak P, Budczies J and Lánczky A: Online survival analysis software to assess the prognostic value of biomarkers using transcriptomic data in non-small-cell lung cancer. PLoS One 8: e82241, 2013.

21. Szklarczyk D, Franceschini A, Kuhn M, Simonovic M, Roth A, Minguez P, Doerks T, Stark M, Muller J, Bork P, et al: The STRING database in 2011: Functional interaction networks of proteins, globally integrated and scored. Nucleic Acids Res 39: D561-D568, 2011.

22. Shannon P, Markiel A, Ozier O, Baliga NS, Wang JT, Ramage D, Amin N, Schwikowski B and Ideker T: Cytoscape: A software environment for integrated models of biomolecular interaction networks. Genome Res 13: 2498-2504, 2003.

23. Bader GD and Hogue CW: An automated method for finding molecular complexes in large protein interaction networks. BMC Bioinformatics 4: 2, 2003.

24. Assenov Y, Ramirez F, Schelhorn SE, Lengauer T and Albrecht M: Computing topological parameters of biological networks. Bioinformatics 24: 282-284, 2008.

25. Luo Y, Xuan Z, Zhu X, Zhan P and Wang Z: Long non-coding RNAs RP5-821D11.7, APCDD1L-AS1 and RP11-277P12.9 were associated with the prognosis of lung squamous cell carcinoma. Mol Med Rep 17: 7238-7248, 2018.

26. Yu T, Zhao Y, Hu Z, Li J, Chu D, Zhang J, Li Z, Chen B, Zhang X, Pan H, et al: MetaLnc9 facilitates lung cancer metastasis via a PGK1-activated AKT/mTOR pathway. Cancer Res 77: 5782-5794, 2017.

27. Liu J, Liu L, Wan JX and Song Y: Long noncoding RNA SNHG20 promotes gastric cancer progression by inhibiting p21 expression and regulating the GSK-3 $\beta / \beta$-catenin signaling pathway. Oncotarget 8: 80700-80708, 2017.

28. Ma C, Wu G, Zhu Q, Liu H, Yao Y, Yuan D, Liu Y,Lv T and Song Y: Long intergenic noncoding RNA 00673 promotes non-small-cell lung cancer metastasis by binding with EZH2 and causing epigenetic silencing of HOXA5. Oncotarget 8: 32696, 2017.

This work is licensed under a Creative Commons Attribution-NonCommercial-NoDerivatives 4.0 International (CC BY-NC-ND 4.0) License. 